Outcomes data of 46 men-the largest series reported to date-were retrospectively evaluated using a validated questionnaire (UCLA/RAND). Prior to surgery, most patients had SUI of moderate severity subsequent to treatment for prostate cancer, and refractory to nonsurgical management. Slingspolypropylene in most cases-were inserted through a perineal incision and anchored at one end to the pubic bone via titanium screws preloaded with polypropylene sutures. Suture tightening generated tension sufficient to compress the bulbous urethra. Tension was optimized during a cough test phase before the second side of the sling was anchored.

Thirty-seven percent of patients reported complete dryness during the average 24-month follow-up period. Symptom severity improved in a further $37 \%$ and overall satisfaction with the sling procedure was $70 \%$. The rate of complication was low; one patient developed an infection, and two required analgesics for perineal/buttock pain. Erosion of the urethra was not reported by any sling recipient.

The authors assert that nonabsorbable slings are a safe and effective additional tool for management of men with SUI. They caution that, until long-term studies prove otherwise, slings should be considered as an alternative to AUS rather than a new standard.

Original article Rajpurkar AD et al. (2005) Patient satisfaction and clinical efficacy of the new perineal bone-anchored male sling. Eur Urol 47: 237-242

\section{Hypnosis helps children through VCUG}

Diagnosis and management of vesicoureteral reflux requires voiding cystourethrography (VCUG). Children with vesicoureteral reflux, in which urine passes retrogradely from bladder to kidney, are typically subjected to four VCUGs. Many find the catheterization, intravesical instillation of contrast media and urination 'on demand' extremely distressing. Memories of this distress can compromise compliance during subsequent VCUGs.

Lisa Butler and co-workers tested whether hypnosis could minimize anxiety. Forty-four children with an average age of 7.6 years, most of whom were white females and had undergone three previous VCUGs, were nonblindly randomized to either hypnosis or routine care prior to their next procedure.

During training in self-hypnosis, children were directed to imagine themselves floating comfortably. They were then instructed to absorb themselves in a competing imaginary situation by visualizing participation in a favorite activity. Parents and children were encouraged to practice this technique several times during each day prior to their next VCUG. Most members of the routine care group elected to participate in a standard recreational therapy session. Sessions focused on familiarizing the child with the phases of VCUG and practicing relaxation. Recreational or hypnosis therapists assisted each child during the procedure.

Hypnosis significantly improved four outcome measures: parental estimation of trauma relative to that experienced by their child during their previous VCUG; typical distress level during the procedure (observed by a research assistant); difficulty of conducting the VCUG (reported by medical personnel); and total procedural time, which was almost 14 minutes less. The authors suggest that hypnosis be used to cost-effectively and noninvasively minimize distress during pediatric VCUG.

Original article Butler LD et al. (2005) Hypnosis reduces distress and duration of an invasive medical procedure for children. Pediatrics 115: e77-e85

\section{Advances in preoperative nodal staging for bladder cancer}

In their recent study, Deserno et al. have shown that ferumoxtran-10-enhanced MRI is superior to nonenhanced MRI in detecting metastatic lymph nodes in patients with bladder cancer. Importantly, this method does not rely on nodal size or shape to distinguish between benign and malignant nodes.

Fifty eight patients with bladder cancer underwent MRI before and 24-36 hours after intravenous injection of ferumoxtran-10 iron oxide nanoparticles. Precontrast images were interpreted on the basis of node size; round nodes larger than $8 \mathrm{~mm}$ or oval nodes of axial diameter greater than $10 \mathrm{~mm}$ were defined as malignant. Postcontrast images were interpreted by comparing them with the precontrast images; benign nodes show a homogenous decrease in signal intensity due 